\title{
PLC-Based Pressure Control in Multi-Pump Applications
}

\author{
Valery Vodovozov (Associate Professor, St. Petersburg Electrotechnical University), Zoja Raud (Associate \\ Professor, Tallinn Technical University), Levon Gevorkov (PhD student, Tallinn University of Technology)
}

\begin{abstract}
The paper is devoted to the centrifugal pumps represented the most popular type of pumping equipment used in different areas. The pressure control approach for variable speed driven (VSD) parallel connected centrifugal pumps is reported. The goal of the study is optimization of some quality indices, such as efficiency, consumed power, productivity, energy carrier temperature, heat irradiation, etc. One of them - efficiency - has been studied in the paper more carefully. The mathematical model of pumping process is discussed and a vector-matrix description of the multi-pump application is given. The programbased pressure control system is developed which productivity is changed by regulating the number of working pumps. The paper introduces new pressure control algorithms based on the working point estimation intended for programmable logical controllers (PLC). Experiments prove correctness of the offered methodology.
\end{abstract}

Keywords - Pumps; Variable speed drives; Pressure control; Power quality; Microcontrollers.

\section{INTRODUCTION}

Pumping plays an important role in liquid delivery, treatment of waste water, fuel production, and other industries [1]. Pumps are applied to transport fluids by converting the rotational kinetic energy of the liquid to the hydrodynamic energy. Centrifugal pumps represent the most popular type of pumping equipment used in different areas and provide from 80 to $90 \%$ of full water treatment [2].

The main purpose of pressure maintenance is to sustain a desired pressure level in a pumping installation or circulation system. Particularly in many applications the liquid being pumped is hot and contains vapour. One of the main advantages of pressure maintenance in such systems is that it prevents phenomena of cavitations at the high points of pipelines. Pressure regulation also compensates unintentional leakages and makes up variations in the volume. Some common cases where the pressure stabilizing is required are the district networks and the water circulation systems in buildings [3].

To control the pump processes, the variable speed drives (VSD) are commonly used that, besides the pressure adjustment, provide a wide range of functionalities including the speed adjustment, technology management as well as the power quality improvement. Programmable logical controllers (PLC) broadly enhance their possibilities.

This paper concentrates on enhancements in the design of the multi-pump systems that support the required pressure level in the pipeline and perform it with such an optimal quality index like the best efficiency. Other quality indices, like maximum productivity, minimum consumed power, optimal energy carrier temperature, optimal heat irradiation, etc. may be studied in the same manner also. A lot of researches in this direction have been published in recent years.

The first group concerns the pressure control. In [4] the model-based pressure monitoring was suggested aiming to detect and partially isolate some faulty pumping conditions. In [5] the feed-forward fuzzy immune algorithm was proposed aiming to tune controllers in the time-varying nonlinear pressure loops. The proportional pressure control in the multivalve heating system with a solo VSD pump was discussed in [6]. A constant pressure supply water station, which adopts embedded PLC-based fuzzy controller, was described in [7]. Using a new fuzzy PID control approach it has been explained in [8] how the collecting pressure performance can be improved with the PLC help. Under invariant control presented in [9], the steam pressure overshoots were decreased in comparison with the traditional PID-feedback control methods.

Different quality metrics were introduced to optimize pumping operation. Most of them are directed to energy saving [10], [11]. In [12], a novel hybrid estimation method for the centrifugal pump operational state was established basing on the process identification by reading the flow rate and head estimates obtained from the manufacturers' characteristics.

The second set of publications has been devoted to the multi-pump plants. The study of the grouping pump operation in the best efficiency region has been conducted in [13] where the adjustment strategy preventing the pumps from operating at low efficiency was based on the VSD online measurements of the flow rate, head, and power of every pump. VSDs without such flow estimation features were excluded from this study. The optimal control of a water boosting system with multiple VSDs in parallel was discussed also in [14] where a framework with combination of feed-forward and feedback controls has been offered. In order to cope with an unknown system operating conditions, enough sophisticated on-line parameter estimation algorithm was proposed in this paper.

In this work, a universal multi-criteria method of the pressure control in the multi-pump stations is proposed and verified basing on such a peculiarity of the PLC architecture like fast operation with pre-defined tabularized data under the limited number of sensors and actuators. The paper is organized as follows. First, the mathematical model of the pumping process is expanded. Then, the vector-matrix model 
of a multi-pump application is described. Among the quality indices of pumping process, an attention is paid to the efficiency estimates in solo and multi-pump applications. After that, a PLC pump control system is described. Finally, the experimental results are presented.

\section{Mathematical Model of PUMPING Process}

Among the variables describing the pumping process the most important are the flow rate $Q$, head $H$, and brake power $P_{\text {shafi }}$ on the pump shaft. Using them, a set of working points of the pump operation is described by the performance traces $(\mathrm{QH}$ characteristics) usually given in the manufacturer documentation for the rated rotational speed. Examples of such traces can be found in [15] and [16].

In the systems designed for the pressure adjustment, the pressure in the pipeline is used as the major control variable found from the Bernoulli's equation [17] as follows:

$$
p=g \rho\left(H-\frac{v^{2}}{2 g}-z\right)
$$

where

$p$ - fluid pressure, $\mathrm{N} / \mathrm{m}^{2}$

$g \rho H-$ initial pressure at the intake, $\mathrm{N} / \mathrm{m}^{2}$

$g \rho z$ - pressure at the measuring point, $\mathrm{N} / \mathrm{m}^{2}$

$p_{0}=g \rho(H-z)-$ static pressure, $\mathrm{N} / \mathrm{m}^{2}$

$\rho \frac{v^{2}}{2}-$ dynamic pressure, $\mathrm{N} / \mathrm{m}^{2}$

$v=\frac{Q}{A}-$ fluid velocity in a pipeline, $\mathrm{m} / \mathrm{s}$

$A$ - cross-sectional area of the pipeline, $\mathrm{m}^{2}$

$\rho$ - fluid density, $\mathrm{kg} / \mathrm{m}^{3}$

$g-$ acceleration due to gravity, $\mathrm{m} / \mathrm{s}^{2}$

$z$-elevation of the point above the reference plane, $\mathrm{m}$

To govern the relationships between the above process variables at the changing pump speed $n$, every hydraulic system is described with the affinity laws [10], [18]:

$$
\begin{gathered}
\frac{Q_{1}}{Q_{2}}=\frac{n_{1}}{n_{2}}, \\
\frac{H_{1}}{H_{2}}=\frac{p_{1}}{p_{2}}=\left(\frac{n_{1}}{n_{2}}\right)^{2}, \\
\frac{P_{1 \text { shaft }}}{P_{2 \text { shaft }}}=\left(\frac{n_{1}}{n_{2}}\right)^{3} .
\end{gathered}
$$

Here, index " 1 " denotes the initial states and index " 2 " - the next states of the variables. With the help of the affinity laws, the family of performance traces may be designed. An example of such a family build with (1), (2), (3) for the Ebara CDX 120/12 pump model [15] is displayed in Fig. 1 with the solid curves of the flow-pressure performance traces of a solo pump at 500,1000, 1500, 1800, 2200, and $2800 \mathrm{rpm}$ respectively.
Beside of the performance traces, every pumping application has its individual system traces that represent the resistance of the pipeline dependent on a specific consumer's design [10]:

$$
p_{s}=p_{0}+k_{p} Q^{2}
$$

where $k_{p}$ is the pressure loss coefficient. In contrast to the performance traces, the system characteristics cannot be provided by manufacturers because they depend on the pipeline topology. Variations of the liquid consumption affect the system curves that tend left as the resistance increases and tend right when the resistance falls. Particularly, at the closed discharge valve the pump pressure is at its maximum and the flow rate approaches almost zero.

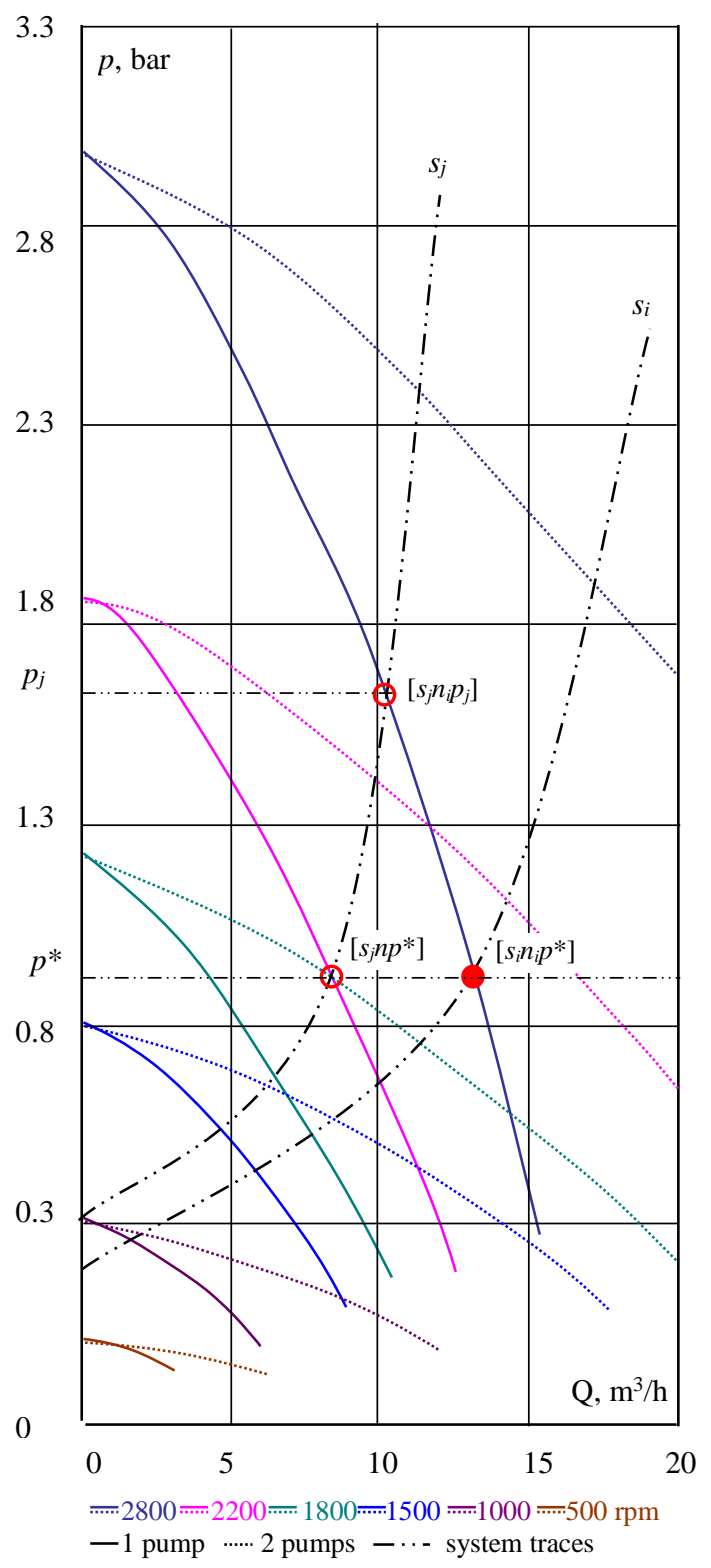

Fig. 1. A family of $Q p$-curves for Ebara CDX 120/12. 
In the VSD-fed pumping applications, the family of the system traces is superimposed upon the family of performance curves. Every intersection of a pump performance trace with the system curve represents some process working point. As an example, in Fig. 1 the family of six performance traces is superimposed by a pair of system traces obtained at different cross-sectional areas with the help of a discharge valve located behind the pump outlet. As a result, a set of 12 working points can be used to discretely describe the pump performance in various working conditions.

\section{VECTOR-MATRIX MODEL OF A MULTI-PUMP APPLICATION}

Let a multi-pump application consists of $R$ pumps. Every pump may rotate at $N$ discrete speed levels $\left\{n_{1}, n_{2}, \ldots, n_{N}\right\}$ supporting $N$ respective performance traces in the $Q p$ reference frame. These traces cross some of the $N$ discrete pressure levels $\left\{p_{1}, p_{2}, \ldots, p_{N}\right\}$. Assume further that the application services $S$ different states described by their system traces $\left\{s_{1}, s_{2}, \ldots, s_{S}\right\}$ in the same $Q p$ reference frame. Enumerate the points of intersections of the performance and system traces as [snp] where $n$ is the speed and $p$ is the nearest pressure level following an intersection point on the $s$ trace. Every [snp]-working point specifies a definite quality index $K_{\text {snp }}$ the pre-defined components of which can be stored in a lookup table of the application PLC. Different quality indices may be used, such as efficiency, consumed power, productivity, energy carrier temperature, heat irradiation, etc [3].

In this way, every possible working state of a pumping process described by its quality index $K_{s n p}$ is represented as a 3-dimensional [snp]-vector (Fig. 2). Each [np]-sub-vector can be described as an $\mathrm{N}$-size squared matrix of the particular system trace at different pressure levels for the respective speeds. Each $[p s]$-sub-vector can be described as an $\mathrm{SN}$-size matrix of the particular speed for different system traces at the respective pressure levels. Each [sn]-sub-vector can be described as an SN-size matrix of the quality indices $K_{s n p}$ at different speed levels for the respective system traces.

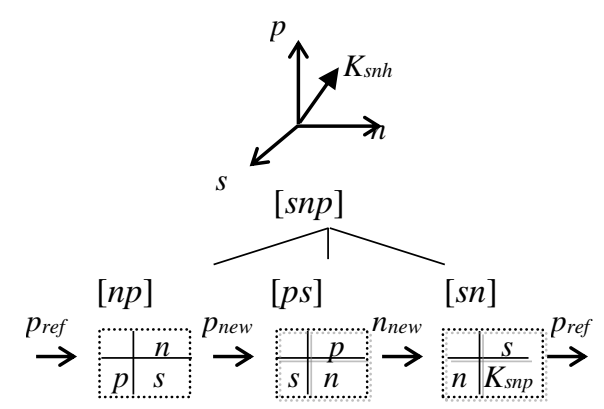

Fig. 2. Vector-matrix model of a pumping application with control steps.

When $r$ identical pumps operate in parallel, their common flow rate is $r$ times higher than the separate pump has. As an example, six dotted lines in Fig. 1 represent the performance traces of a pair of parallel-connected pumps.

The model of $R$ parallel connected identical pumps is represented by $R$ similar vectors those flow rates for every $r$ pumps are $r$ times higher than in the separate pump model. The number of system traces $S$ can now be increased.

\section{EFFICIENCY AS A QUALITY INDEX OF PUMPING PROCESS}

The trace intersections [snp] are placed in the application operating regions described with their quality indices $K_{\text {snp }}$. In many applications [11]-[14], the efficiency is assigned as a major quality index. The pumping application efficiency is as follows:

$$
\eta=\frac{P}{P_{\text {sup }}}=\eta_{P} \eta_{M} \eta_{C}=\frac{P}{P_{\text {shaft }}} \frac{P_{\text {shaft }}}{P_{C}} \frac{P_{C}}{P_{\text {sup }}}
$$

where

$$
\begin{aligned}
& P=g \rho Q H-\text { hydraulic power, } \mathrm{W} \\
& P_{\text {sup }}-\text { electrical supply power, } \mathrm{W} \\
& P_{\text {shaft }}-\text { brake power on the motor shaft, } \mathrm{W} \\
& P_{C}-\text { electrical power of the converter, W } \\
& \eta_{P}-\text { efficiency of the pump } \\
& \eta_{M}-\text { efficiency of the motor } \\
& \eta_{C}-\text { efficiency of the power converter }
\end{aligned}
$$

To estimate efficiencies in the application working points, the following algorithm is proposed.

From the manufacturer documentation, obtain the diagram of the brake power versus the flow rate which is usually given along with the performance traces for the rated speed. Basing on these power traces and using the affinity law (4), find the brake power $P_{\text {shaft }}$ in every working point. As well, the mechanical torques $T$ on the pump motor shaft can be defined as follows [19], [20]:

$$
T=\frac{P_{\text {shaft }}}{\omega}
$$

where

$$
\begin{aligned}
& T \text { - torque on the motor shaft, } \mathrm{Nm} \\
& \omega=\frac{2 \pi n}{60}-\text { angular frequency, } \mathrm{rad} / \mathrm{s}
\end{aligned}
$$

After that, address the drive manufacturers' documentation, for example [21], [22], where the motor and power converter losses $\Delta P_{M C}$ are collected for their part-torque operation at different speeds. Using (6), the efficiencies in the application working points $\eta$ can be estimated as follows:

$$
\eta=\eta_{P} \eta_{M} \eta_{C}=\frac{P}{P_{\text {shaft }}} \cdot \frac{P_{\text {shaft }}}{P_{\text {shaft }}+\Delta P_{M C}}
$$

For example, in Fig. 3 a family of $Q p$-curves developed for the solo Ebara CDX 120/12 is superimposed by some efficiency regions, from less than 25 to more than $38 \%$ (solid lines). To build them, the pump efficiencies ranged from 30 to $48 \%$ were acquired from [15] whereas the motor drive efficiencies ranged from 5 to $78 \%$ were acquired from [22].

In the case of $r$ pumps, their common brake power and drive losses increase $r$ times compared to the separate one. In Fig. 3 a family of $Q p$-curves for the pair of parallel-connected Ebara CDX $120 / 12$ is superimposed by the appropriate efficiency regions (dotted lines). 
The same approach can be applied for estimation of such a quality index as minimum consumed power. Other quality indices can be explored as well.

\section{PUMP CONTROL USING PLC}

Let the primary goal of the control be to stabilize some reference pressure at variable system states and the secondary goal - to achieve one of the quality indices. Multiple control algorithms can be offered using the above model.

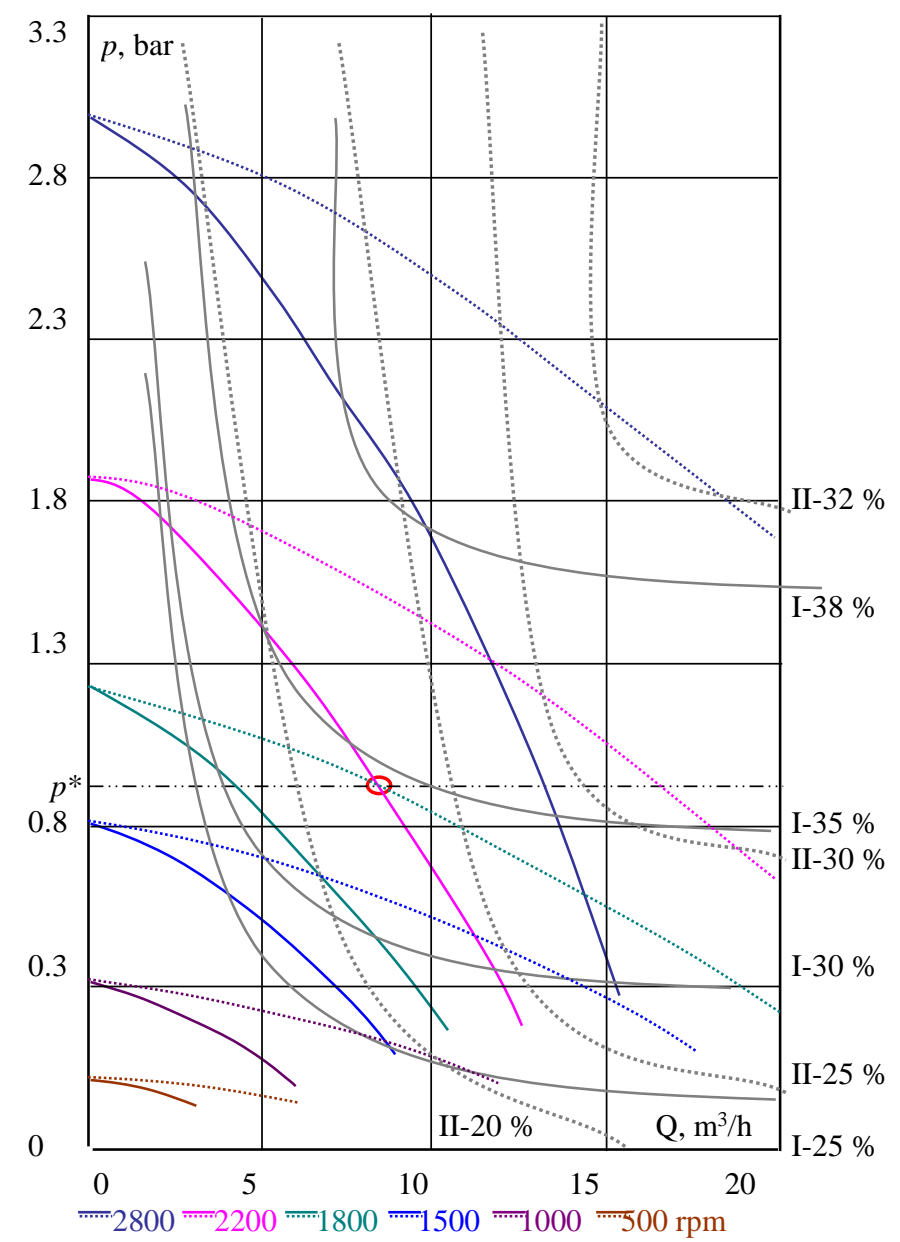

Fig. 3. A family of $Q p$-curves for the solo Ebara CDX 120/12 (solid lines) and the pair of parallel-connected pumps (dotted lines) driven by ACQ810 VSD with appropriate efficiency regions for the solo (I) and paired (II) pumps.

If the designer explores all the model variables, the simulation algorithm will be as follows. Let initially the pumping application be in $i$-th working point $\left[s_{i} n_{i} p^{*}\right]$ shown in Fig. 1 . It means that $r(r \in R)$ pumps rotating at $n_{i}$ speed each support a reference pressure $p^{*}$ along some system trace $s_{i}$. Assume that under the influence of a disturbance the pressure has been changed to $p_{j}$ and called the working point transition to some new system trace $s_{j}$ at the same speed $n_{i}$. The control task is to shift the working point to the new position $\left[s_{j} n_{x} p^{*}\right]$ of the system trace $s_{j}$ to support the reference pressure level $p^{*}$.

To this aim, once the sensor detects the new pressure $p_{j}$, the PLC will search the appropriate system trace $s_{j}$ at the intersection of $p_{j}$ row and $n_{i}$ column of the $[n p]$-matrix in Fig. 2. Since the new system trace is known, the search of the required $n_{x}$ speed will start at the intersection of the $s_{j}$ row and $p^{*}$ column of the $[p s]$-matrix. An intersection of the $n_{x}$ row and $s_{j}$ column of the $[s n]$-matrix will indicate the quality index $K_{\text {snp }}$.

In the $R$-pump application, $R[p s]$-matrixes are to be observed aiming to find $R$ different pump speeds of parallelconnected pumps. Every variant will indicate its own quality index $K_{\text {snp }}$. These quality indices are to be compared aiming to choose the variant with the highest one. In some cases a single high-speed pump represents the best choice but in other cases two or three low-speed pumps will produce better quality metric of the application.

Often, the only designer's target is to find the numbers and speeds of the parallel connected pumps irrelative of the system traces. In this case the following problem is stated. Let, under a disturbance, the application has appeared at some pressure $p_{j}$ of the performance trace $n_{j}$. The control task is to find the optimal number of pumps $r$ and their speeds $n$ to support the reference pressure level $p^{*}$. Now, it is enough to use only $R$ [np]-matrixes where the quality indices $K_{s n p}$ are located at the intersections of $p^{*}$ rows and $n$ columns. The speed under the question can be calculated using the affinity law (3) as follows:

$$
n=n_{i} \sqrt{\frac{p^{*}}{p_{j}}} .
$$

As soon as the PLC finds the appropriate quality indices $K_{\text {snp }}$, they have to be compared and the variant with the best one is chosen. Once the solution found, the required pumps are running, their reference pressures assigned, and the pressure control executed.

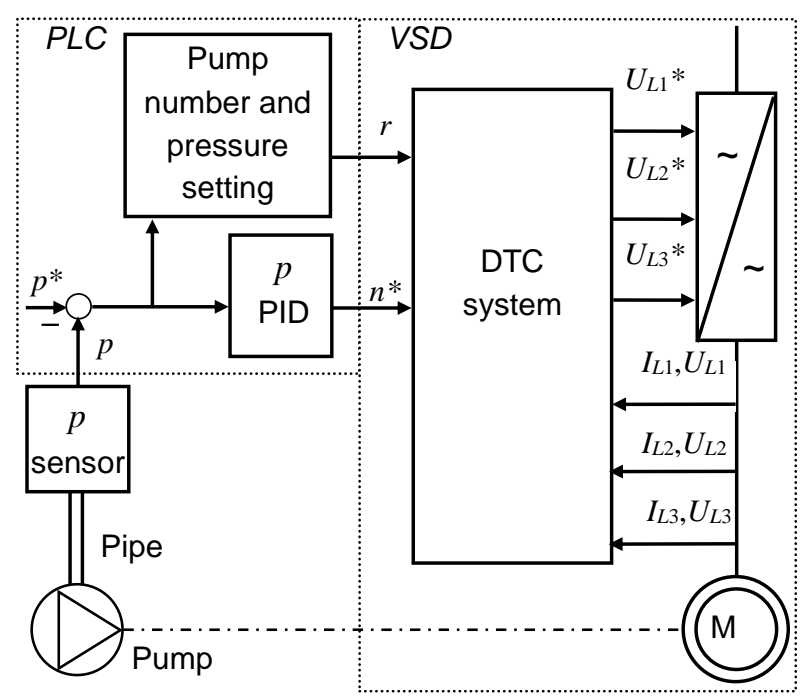

Fig. 4. Functional diagram of program-based pressure control.

In the functional diagram in Fig. 4 an implementation example is proposed.

An $R$-pump application consists of the common PLC connected to $R$ VSDs one of those is shown. The PLC realises the control algorithm and runs $r$ required pumps. A motor $\mathrm{M}$ 
connected to the pump is supplied with a power electronic converter. The direct torque control (DTC) system acquires the motor alternating currents $I_{L 1}, I_{L 2}, I_{L 3}$ and voltages $U_{L 1}$, $U_{L 2}, U_{L 3}$ using the appropriate sensors and converts them to the direct current and voltage signals. Using the reference speed $n^{*}$, the DTC system identifies the desired supply voltages $U_{L 1} *, U_{L 2} *, U_{L 3} *$ for the power converter to run the pump.

The reference speed $n^{*}$ of every VSD is received from the output of the proportional-integral-differential (PID) pressure regulator which estimates, restricts, and converts the difference of the reference $p^{*}$ and actually measured $p$ pressures.

Any time when, under the influence of a disturbance, the pressure changes calling the working point transition to some new system trace, the PLC runs the above proposed algorithm aiming to find the best choice from the viewpoint of the quality index, is it a single pump rotating at some recommended speed or several pumps working at lower speeds. After that, the required pumps are running and the pressure PID control executed. The pressure control accuracy is defined by the resolution of the PID regulator, its algorithm as well as the VSD parameters.

For example, let the target working point is [ $\left.s_{j} n p^{*}\right]$ in Fig. 1 where two variants of control are possible: one pump operated at the speed $2200 \mathrm{rpm}$ or two pumps operated at the speeds $1800 \mathrm{rpm}$ each. As follows from Fig. 3, in the former case the efficiency is about $34 \%$. As follows from Fig. 4, in the latter case the efficiency is about $23 \%$. Hence, from the efficiency point of view one pump operated at higher speed better suits the process quality.

\section{EXPERIMENTATION}

The aim of experimentation was to provide a valid pressure control. At the appropriate pressure control, the offered algorithm may be executed applying the PLC programming. An ABB manufactured experimental pumping station has been used in this study. The application is accomplished with five centrifugal pumps Ebara 120/12 $(0.9 \mathrm{~kW}, 400 \mathrm{~V}, 3 \mathrm{~A}$, $2800 \mathrm{rpm}$ ) driving by the ACQ810 VSDs, discharge valves, a pressure sensor and manometer, digital switches, potentiometers, relay and data cable circuitry, for RS485 connection. The station includes all the required hardware and software to adjust the pumping speed with process monitoring. The toolkit Drive Studio provides the distance control, data acquisition, and collection. To explore different system curves, the operator can change the pipe cross-sectional area using a discharge valve. Detailed description of the composition of the experimental setup can be found in [23].

As a PLC, AC500 PLC series has been chosen. For communication, Ethernet, Profibus DP, Modbus TCP, Modbus serial and similar modules may be used. AC500 connections with computer, ACQ810 VSDs, and the pumping station is shown in Fig. 5.

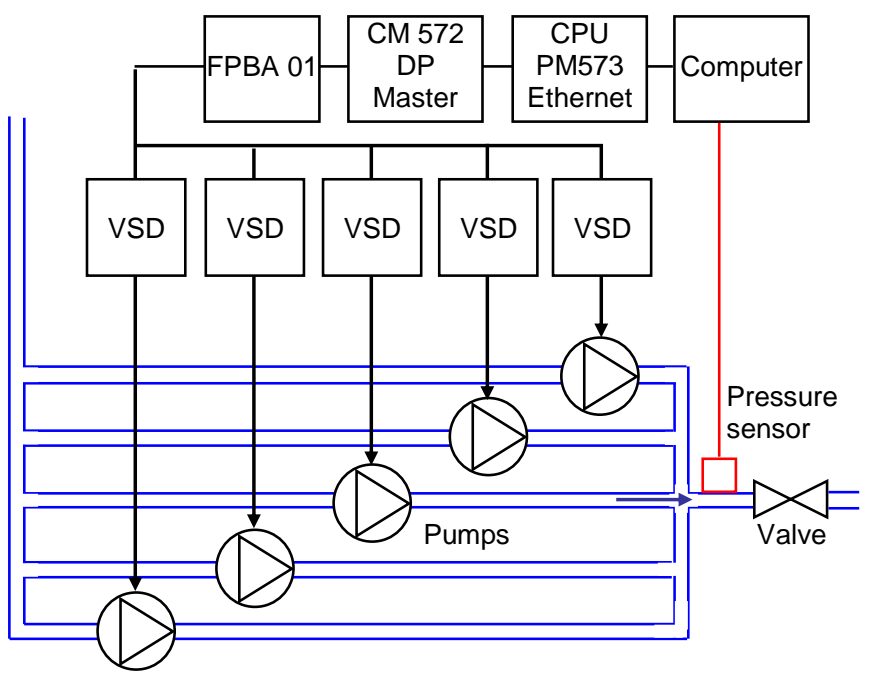

Fig. 5. Topology of the 5-pump station.

Here,

CM572 DP - communication module Profibus-DP Master, PM573 - PLC interface module,

FPBA-01 - Profibus DP adapter module.

The ACQ810 VSDs are connected via the network cable to the FPBA-01 DP adapter module. The designed interface transmits commands to the VSDs and receives data related to working mode of the pumping system. Productivity of the system is changed by regulating the number of working pumps which should be increased to raise the pressure. Unnecessary pumps can be stopped if the actual pressure is higher than required. Generation of the speed reference and regulation of the number of working pumps is conducted by one of the VSDs called a master of the network. In this topology the control system serves the master drive and the rest of VSDs are slaves. In the conflict situations like those shown in $\left[s_{j} n p^{*}\right]$ point in Fig. 1, the PLC comes to a decision accordantly the above algorithm. If the number of the required pump overcomes the equipment limit, the pumping will stop.

In the study the ABB Automation Builder has been used. This toolkit combines all the instruments needed to configure, program, debug, and collect common data and maintenance the automation projects using an intuitive interface with broad functionality. Additionally, the control panel has been assembled with 16 on/off switches, two resistive potentiometers, and a voltage measuring device. The software was prepared in Controller Development System within the 3S-Smart Software Solutions (CODESYS) V2.3 environment [24], [25], [26]. CODESYS combines such programming tools as instruction language, structural text language, ladder diagram language, function block diagrams, sequential function charts. During the experiments an angle of the discharge valve installed in the pipeline was changed discretely as shown in Fig. 6. 


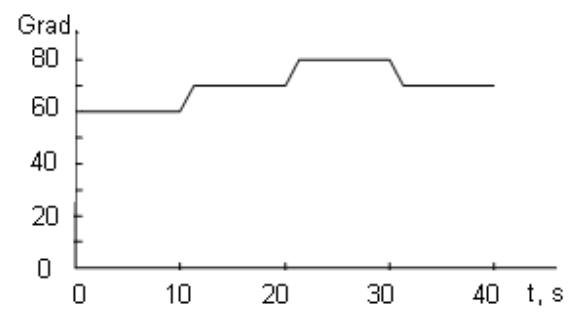

Fig. 6. Valve angle regulation diagram.

An example of the system response is shown in Fig. 7.

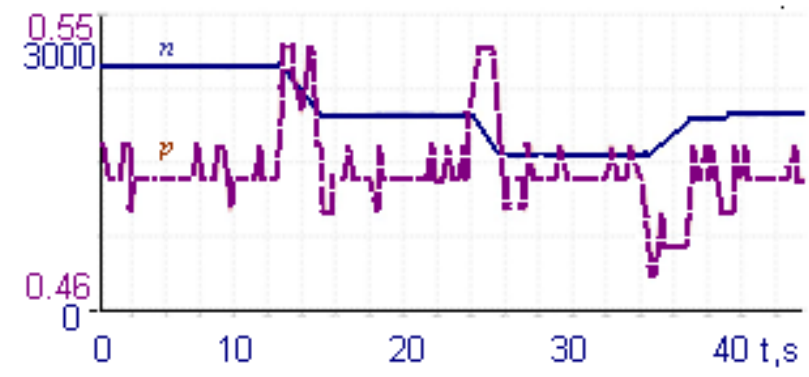

Fig. 7. Experimental pressure and speed responses.

As follows from the experiments, once the system curve rushes left (valve closing in Fig. 6), the working point moves up along the pump performance characteristic thus increasing the pressure. As soon as the sensor feels the pressure growth, the pressure PID controller decreases the reference speed motivating the DTC control system to set the reduced voltage for the power converter. As a result, the motor speed drops stabilizing the pressure on the desired set-point level as shown in Fig. 7. And vice versa, if the system curve rushes right (valve opening in Fig. 6), the working point moves down decreasing the pressure. Once the sensor feels the pressure lowering, the PID controller increases the reference speed motivating the DTC to enlarge voltage of the power converter. As a result, the motor speed grows thus stabilizing the pressure on the desired level.

\section{CONCLUSION}

The new pressure control algorithms based on vector-matrix working point estimation intended for PLC-based pumping applications have been proposed and studied verified. Appropriate software for the multi-pump and multi-drive installation was developed and verified. A possibility to handle the pressure was experimentally tested. Besides effective pressure regulation the developed system provides the plant operation in the best efficiency regions and may support other quality indices, like maximum productivity, minimum consumed power, optimal energy carrier temperature, optimal heat irradiation, etc.

\section{ACKNOWLEDGMENT}

This research work has been supported by the Estonian Ministry of Education and Research (Project SF0140016s11)

\section{REFERENCES}

[1] H. L. Stewart, Pumps, Indianapolis: Sams, 1977, 465 p.

[2] G. Hovstadius, V. Tutterow and S. Bolles, "Getting it right. Applying a system approach to variable speed pumping," in 4th Int. Conf. on Energy Efficiency in Motor Driven Systems, EEMODS 2005, Heidelberg, Germany, 2005, pp. 304-314.

[3] B. Nesbitt, Handbook of Pumps and Pumping, Elsevier, 2006, 424 p.

[4] H.-Z. Tan and N. Sepehri, "On condition monitoring of pump pressure in a hydraulic servo-drive system," in Proc. of the 2001 American Control Conf., Arlington, VA, 2001, pp. 4478-4483. http://dx.doi.org/10.1109/ACC.2001.945684

[5] Y. Ji, S. Peng, L. Geng, Z. Wang and L. Qiu, "Pressure loop control of pump and valve combined EHA based on FFIM", in The 9th Int. Conf. on Electronic Measurement \& Instruments, ICEMI 2009, Beijing, China, pp. 3-578-3-582. http://dx.doi.org/10.1109/icemi.2009.5274250

[6] J. Tamminen, T. Ahonen, A. Kosonen, J. Ahola and J. Tolvanen, "Variable speed drive-based pressure optimization of a pumping system comprising individual branch flow control elements," in 16th European Conf. on Power Electronics and Applications, EPE 2014-ECCE Europe, Lappeenranta, Finland, 2014 pp. 1-11. http://dx.doi.org/10.1109/EPE.2014.6910988

[7] P. Xiaohong, X. Laisheng, M. Zhi and L. Guodong, "The variable frequency and speed regulation constant pressure water supply system based on PLC and fuzzy control," in Int. Conf. on Measuring Technology and Mechatronics Automation, ICMTMA 2009, Zhangjiajie, Hunan, 2009, pp. 910-913. http://dx.doi.org/10.1109/ICMTMA.2009.392

[8] C. Wei1, X. Meixiang and F. Kangling, "A PLC-based fuzzy PID controller for pressure control in coke-oven," in 31st Chinese Control Conference, CCC, Hefei, China, 2012, pp. 4754-4758.

[9] A. Sniders and T. Komass, "Invariant method of load independent pressure control in steam boiler," Electrical, Control and Communication Engineering, vol. 1, issue 1, 2012, pp. 5-10. http://dx.doi.org/10.2478/v10314-012-0001-4

[10] R. Carlson, "The correct method of calculating energy savings to justify adjustable-frequency drives on pumps," in IEEE Trans. on Ind. Appl., vol. 36, no. 6, 2000, pp. 1725-1733. http://dx.doi.org/10.1109/28.887227

[11] L. Szychta and R. Figura, "Analysis of efficiency characteristics of squirrel-cage induction motor for pump applications," in 20th Int. Conf. on Electrical Machines, ICEM 2012, Marseille, France, 2012 pp. 73-78. http://dx.doi.org/10.1109/icelmach.2012.6349842

[12] T. Ahonen, J. Tamminen, J. Ahola and J. Kestilä, "Frequency-converterbased hybrid estimation method for the centrifugal pump operational state," IEEE Trans. on Ind. Electron., vol. 59, no. 12, 2012, pp. 48034809. http://dx.doi.org/10.1109/TIE.2011.2176692

[13] J. Viholainen, J. Tamminen, T. Ahonen, J. Ahola, E. Vakkilainen and R. Soukka, "Energy-efficient control strategy for variable speed-driven parallel pumping systems," in Energy Efficiency, vol. 6, 2013, pp. 495509. http://dx.doi.org/10.1007/s12053-012-9188-0

[14] Z. Yang and H. Børsting, "Energy efficient control of a boosting system with multiple variable-speed pumps in parallel," in 49th IEEE Conf. on Decision and Control, Atlanta, GA, USA, 2010, pp. 2198-2203. http://dx.doi.org/10.1109/CDC.2010.5717312

[15] Centrifugal Pumps CDX, Available at: http://ebara-pumpsonline.com/CDX.pdf.

[16] Danfoss Products and Solutions, Available: http://www.danfoss.com.

[17] J. E. Finnemore and J. B. Franzini, Fluid Mechanics with Engineering Applications, NY: McGraw Hill, Inc., 2002, 500 p.

[18] I. I. Ionel, Pumps and Pumping with Particular Reference to VariableDuty Pumps, Amsterdam: Elsevier, 1986, 715 p.

[19] P. G. Kini, R. C. Bansal and R. S. Aithal, "Performance analysis of centrifugal pumps subjected to voltage variation and unbalance," IEEE Trans. on Ind. Electron., vol. 55, no. 2, 2008, pp. 562-569. http://dx.doi.org/10.1109/TIE.2007.911947

[20] V. Vodovozov, Electrical Drive: Performance, Design and Control, Lambert Academic Publishing, Saarbrücken, 2014, $320 \mathrm{p}$.

[21] ABB Oy Efficiency Estimation Guide, Available: http://www05.abb.com/global/scot/scot201.nsf/veritydisplay/ab22cf21c3 67d260c12573e9004d88a4/\$file/efficiency_calc_guide.pdf.

[22] DriveSize, Available: http://ds.nodus.info/starter.aspx.

[23] V. Vodovozov and I. Bakman, "Performance improvement of pumps fed by the variable-speed drives," Electrical, Control and Communication Engineering, vol. 4, issue 1, 2013, pp. 45-50. http://dx.doi.org/10.2478/ecce-2013-0021 
[24] CODESYS in Embedded Automation: Complete IEC 61131-3 IDE for industrial embedded applications, Available: http://www.codesys.com/.

[25] Codesys, Cel Publishing, 2012, 96 p.

[26] B. Vogel-Heuser, Automation \& Embedded Systems, Oldenbourg Industrieverlag, 2008, $78 \mathrm{p}$.

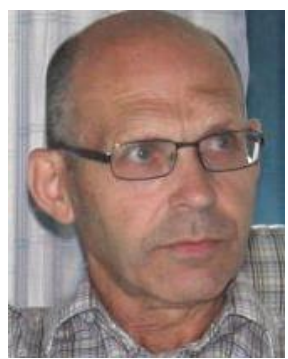

Valery Vodovozov received his Candidate of Science degree and Associate Professor and Senior Researcher academic titles in Electrical Engineering from St. Petersburg Electrotechnical University, Russia. He is a professor of Tallinn University of Technology, a member of IEEE, International Institute of Informatics and Systemics (IIIS), and Global Research Alliance of Texas Institute of Science (TxIS GRA)

Postal address: Ehitajate tee 5, Tallinn, 19086, Estonia.

E-mail: valery.vodovozov@ttu.ee

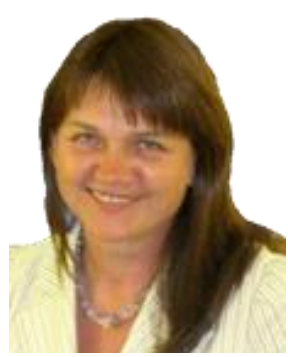

Zoja Raud received education in Tallinn Technical University and received her PhD degree in the field of electrical drives and power electronics from the same university. She is an associated professor of Department of Electrical Engineering where she taught academic courses in Electronics and Power Electronics.

Postal address: Ehitajate tee 5, Tallinn, 19086 Estonia.

E-mail: learnelectronics@narod.ru

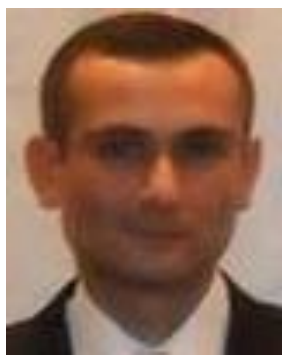

Levon Gevorkov was educated at the Ivane Javakhishvili State University of Tbilisi, where he received the B.S. and M.S. degrees in radiophysics and electronics, in 2001 and 2003, respectively. Currently, he is a $\mathrm{PhD}$ student of Tallinn University of Technology. Main scientific interests lay in the field of electrical drives and pumping systems.

Postal address: Ehitajate tee 5, Tallinn, 19086,

Estonia.

E-mail: learndrives@gmail.com 